\title{
Ventilação Mecânica, Desmame Ventilatório e Qualidade do Sono em UTI: Revisão Sistemática
}

\author{
Mechanical Ventilation, Ventilatory Weaning and Sleep \\ Quality in ICU: Systematic Review
}

\author{
Ventilación Mecánica, Residuos Ventiladores y Calidad del \\ Sueño em UTI: Revisión Sistemática
}

\author{
Bruna Antoniassi Marchezini ${ }^{1}$; Maria Laura Fernandes Gaigher ${ }^{2}$; \\ Izabela Moreira Bomfim 3 ; Alana Kock Ferreguetti Costa ${ }^{4}$
}

\begin{abstract}
1.Fisioterapeuta pela Universidade Federal do Espírito Santo (UFES). Residente Multiprofissional em Cardiologia pelo Hospital Evangélico de Vila Velha (HEVV)/ Vila Velha-ES, Brasil.

2.Fisioterapeuta pela Escola Superior de Ciências da Santa Casa de Misericórdia de Vitória (EMESCAM). Residente Multiprofissional em Cardiologia pelo Hospital Evangélico de Vila Velha (HEVV)/ Vila Velha-ES, Brasil.

3. Fisioterapeuta pela Universidade Federal do Espírito Santo (UFES). Residente Multiprofissional em Cardiologia pelo Hospital Evangélico de Vila Velha (HEVV)/ Vila Velha-ES, Brasil.

4. Fisioterapeuta pela Universidade de Vila Velha (UVV). Especialista em Cardiologia pelo programa de Residência Multiprofissional de Cardiologia da Universidade Federal de São Paulo / Fisioterapeuta contratada do Hospital Evangélico de Vila Velha (HEVV), Vila Velha-ES, Brasil.
\end{abstract}

\begin{abstract}
Resumo
Introdução. As anormalidades do sono durante a internação em unidade de terapia intensiva são bem estabelecidas. Pacientes críticos apresentam deterioração do sono. A ventilação mecânica também pode causar interrupção do sono nesta população, causando a redução das fases restauradoras do sono. Acredita-se que as assincronias paciente-ventilador são responsáveis pela deterioração da qualidade do sono, gerando apneias centrais e despertares. Estratégias não farmacológicas para a melhoria da qualidade do sono nesta população incluem ajustes ventilatórios bem como do próprio modo ventilatório. Objetivo. avaliar as estratégias não farmacológicos, que propiciem uma melhor qualidade de sono em pacientes críticos. Método. Foram utilizadas as bases de dados: Pubmed, Lilacs e Medline. Resultados. Nove ensaios clínicos foram selecionados segundo os critérios de inclusão e exclusão, publicados entre 2007 a 2017. Conclusão. A monitorização do sono na UTI é dispendiosa e trabalhosa. Os distúrbios do sono trazem prejuízos fisiológicos importantes, a seleção do modo ventilatório e ajuste pormenorizado personalizado dos parâmetros ventilatórios utilizados durante o período de sono são essências para melhora do sono. Os novos modos ventilatórios como NAVA e PAV+ auxiliam nesses ajustes. Além disso a adequação do ambiente também é uma estratégia não farmacológica a ser considerada.
\end{abstract}

Unitermos. Ventilação mecânica; polissonografia; qualidade do sono; cuidados críticos

\begin{abstract}
Introduction. Abnormalities of sleep during intensive care unit stay are well established. Critical patients present with sleep deterioration. Mechanical ventilation can also cause sleep disruption in this population, causing the reduction of sleep restorative phases. It is believed that patient-ventilator asynchronies are responsible for the deterioration of sleep quality, generating central apneas and awakenings. Non-pharmacological strategies for improving sleep quality in this population include ventilatory adjustments as well as the ventilatory mode itself. Objective. to evaluate non-pharmacological strategies that provide better sleep quality in critically ill patients. Methods. The databases were Pubmed, Lilacs, and Medline. Results. Nine clinical trials were selected according to the inclusion and exclusion criteria published between 2007 and 2017. Conclusion. Sleep monitoring in the ICU is expensive and toilful.
\end{abstract}


Sleep disturbances causes important physiological disorders, and choosing the right ventilatory mode and detailed personalized adjustment of the ventilatory parameters are essential for improving sleep quality. New ventilation modes such as NAVA and PAV + could help with this improvements. In addition, the adequacy of the environment is also a non-pharmacological strategy to be considered.

Keywords. Mechanical ventilation; Polysomnography; Sleep quality; critical care

\section{RESUMEN}

Introducción. las anormalidades del sueño durante el ingreso a la unidad de cuidados intensivos están bien establecidas. Los pacientes críticos tienen deterioro del sueño. La ventilación mecánica también puede causar trastornos del sueño en esta población, lo que provoca una reducción en las fases restaurativas del sueño. Se cree que los asíncronos del paciente-ventilador son responsables del deterioro de la calidad del sueño, generando apnea central y despertar. Las estrategias no farmacológicas para mejorar la calidad del sueño en esta población incluyen ajustes ventilatorios, así como el propio modo ventilatorio. Objetivo. evaluar las estrategias no farmacológicas que proporcionan una mejor calidad del sueño en pacientes críticos. Método. Se utilizaron las siguientes bases de datos: Pubmed, Lilacs y Medline. Resultados. Se seleccionaron nueve ensayos clínicos de acuerdo con los criterios de inclusión y exclusión, publicados entre 2007 y 2017. Conclusiones. la monitorización del sueño en la UCI es costosa y laboriosa. Las alteraciones del sueño traen importantes alteraciones fisiológicas, la selección del modo de ventilación y el ajuste detallado personalizado de los parámetros de ventilación utilizados durante el sueño son esenciales para la mejora del sueño. Nuevos modos de ventilación como NAVA y PAV + ayudan con estos ajustes. Además, la idoneidad ambiental también es una estrategia no farmacológica a tener en cuenta.

Palabras clave: ventilación mecânica; polisomnografía; calidad del sueño; cuidados críticos

Trabalho realizado no Hospital Evangélico de Vila Velha, Vila Velha-ES, Brasil.

Endereço para correspondência: Alana KF Costa. Av. Saturnino Rangel, 3302, Vila Velha-ES, Brasil. E-mail: alanakock@hotmail.com

\section{INTRODUÇÃO}

As anormalidades do sono durante a internação em unidade de terapia intensiva (UTI) são conhecidas há décadas ${ }^{1}$. Pacientes gravemente doentes apresentam as fases do sono desorganizadas, e de má qualidade. Em ambiente hospitalar estes pacientes precisam enfrentar inúmeros obstáculos ao sono, como por exemplo, as características da própria doença, os fatores estressores presentes no ambiente, a movimentação da equipe, e as intervenções realizadas durante o período da noite ${ }^{2-4}$. 
Estudos prévios demonstraram que a ventilação mecânica também pode causar interrupção do sono em pacientes críticos, apesar dos mecanismos responsáveis por essa interrupção ainda não serem totalmente definidos ${ }^{4,5}$.

Indivíduos mecanicamente ventilados apresentam um predomínio do estágio $\mathrm{N} 1$ da fase de movimento não rápido dos olhos (NREM), redução significativa da fase N3 do NREM e da fase de Movimento Rápido dos Olhos (REM). O que configura que o paciente permanece uma menor quantidade de tempo no estágio onde ocorre o sono reparador ${ }^{5,4}$. Estes pacientes apresentam um padrão polissonográfico alterado, onde não estão presentes as fases típicas do sono, dando lugar a um fenômeno chamado "vigília patológica"3. Alguns autores afirmam que as assincronias paciente-ventilador são responsáveis por um grau de deterioração da qualidade do sono, gerando apneias centrais e fragmentação ${ }^{6}$. Para melhorar esta interação, novos modos ventilatórios foram propostos e sua influência na qualidade do sono são pouco estudadas.

Um sono de má qualidade e não restaurador em ambiente de terapia intensiva pode gerar uma série de consequências ao indivíduo como: depressão, ansiedade, redução do limiar de dor, disfunção cognitiva, redução de resistência da musculatura respiratória, redução da sensibilidade á hipóxia e hipercapnia, aumento do tônus simpático, aumento de interleucinas 6 e proteínas C-reativa, aumento do metabolismo, e redução da imunidade ${ }^{7-11}$. 
Neste contexto estratégias não farmacológicas para a melhoria da qualidade do sono em pacientes de terapia intensiva incluem ajustes ventilatórios bem como do próprio modo ventilatório, que ofereçam um sono restaurador. Diante disso, o objetivo do presente estudo foi avaliar as estratégias não farmacológicos, com foco nos ajustes ventilatórios, que propiciem uma melhor qualidade de sono em pacientes críticos mecanicamente ventilados.

\section{MÉTODO}

Trata-se de uma revisão sistemática, onde foi realizada a busca nas bases de dados Pubmed, Lilacs, e Medline, utilizando-se os descritores "Mechanical ventilation", "Polysomnography", "Sleep quality", "critical care", pesquisados isoladamente e em cruzamento, no período de setembro de 2018 a janeiro de 2019, e limitando-se apenas aos artigos escritos em língua inglesa. Além disso, para complementar, foi realizada uma busca manual nas referências dos artigos selecionados para análise. Foram incluídos todos os artigos que estivessem disponíveis na íntegra com livre acesso, publicados no período de 2007 a 2017, versando sobre a temática proposta. Foram excluídos os artigos levantados nas bases de dados com o uso dos descritores propostos, mas que versam sobre temática diferente do tema proposto, além dos artigos repetidos e as revisões. 


\section{RESULTADOS}

Um total de 76 artigos foram encontrados nas bases de dados selecionadas. Na pré-avaliação dos títulos e resumos, foram selecionados para análise 6 artigos. Depois da busca manual nas referências destes 6 artigos, foram incluídos mais 3 para análise aprofundada. Os demais artigos foram excluídos, levando em consideração os critérios de inclusão e exclusão estabelecidos.

Cinco artigos ${ }^{5,6,12-14}$ eram estudos cruzados comparativos e prospectivos e os demais 4 artigos $^{2,15-17}$ eram estudos cruzados randomizados. O processo detalhado para aquisição dos estudos encontra-se na Figura 1. Um total de 146 indivíduos foram estudados, e as suas características estão descritas na Tabela 1 . O detalhamento dos artigos se encontra na Tabela 2.

\section{DISCUSSÃO}

A redução da duração das fases restauradoras do sono, ou até mesmo abolição dessas fases, o aumento exacerbado do número de fragmentações do sono e vigília patológica são achados recorrentes nos estudos sobre qualidade do sono em pacientes mecanicamente ventilados ${ }^{18-21}$. Um estudo recente, demonstrou que o tempo de desmame ventilatório de pacientes que já apresentam desmame difícil, pode ser ainda maior naqueles que apresentam padrões de sono atípico $^{22}$. 


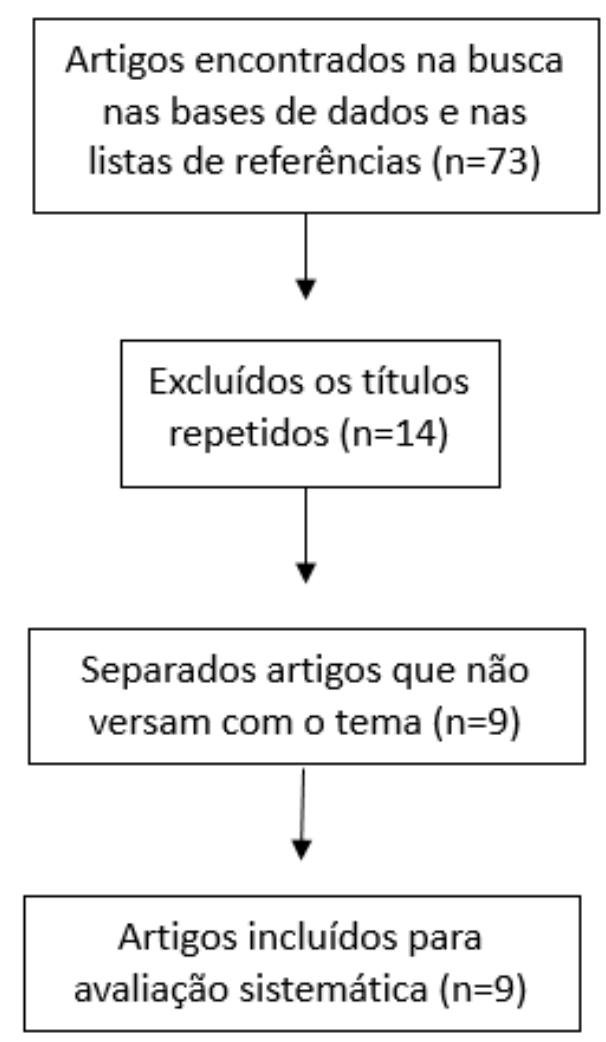

Além disso, a privação do sono pode levar a alterações na resposta ventilatória ao aumento do CO2 e à hipóxia, além de reduzir a resistência da musculatura respiratória, que também é uma causa de falha no desmame ventilatório prolongando o tempo de ventilação mecânica10,23.

Pacientes traqueostomizados que se encontravam em processo de desmame ventilatório, foram comparados quanto a qualidade do sono em respiração espontânea com suplementação de $\mathrm{O} 2$ e em ventilação mecânica em modo Ventilação com pressão de suporte (PSV) ${ }^{15}$. Observaram que permanecer períodos em ventilação mecânica a noite não interferiu no processo de desmame, e não foi observado diferença na qualidade do sono entre a ventilação mecânica 
Tabela 1. Perfil dos pacientes estudados.

\begin{tabular}{|c|c|c|c|c|}
\hline Estudo & Pacientes & Sexo & $\begin{array}{l}\text { Média } \\
\text { de } \\
\text { idade }\end{array}$ & $\begin{array}{l}\text { Motivo da } \\
\text { ventilação } \\
\text { mecânica }\end{array}$ \\
\hline $\begin{array}{l}\text { Alexopoulou et } \\
\text { al., } 2013^{2}\end{array}$ & $\begin{array}{l}14 \text { pacientes não } \\
\text { sedados }\end{array}$ & $\begin{array}{l}9 \text { homens } \\
4 \text { mulheres }\end{array}$ & 72 anos & $\begin{array}{l}\text { Infecção } \\
\text { pulmonar/ } \\
\text { Exacerbação da } \\
\text { DPOC }\end{array}$ \\
\hline $\begin{array}{l}\text { Cabello et al., } \\
2008^{5}\end{array}$ & $\begin{array}{l}15 \text { pacientes não } \\
\text { sedados }\end{array}$ & $\begin{array}{l}11 \text { homens } \\
4 \text { mulheres }\end{array}$ & 70 anos & $\begin{array}{l}\text { Insuficiência } \\
\text { cardíaca/ EAP }\end{array}$ \\
\hline $\begin{array}{l}\text { Delisle et al. } \\
2011^{6}\end{array}$ & $\begin{array}{l}14 \text { pacientes não } \\
\text { sedados }\end{array}$ & $\begin{array}{l}8 \text { homens } \\
6 \text { mulheres }\end{array}$ & 64 anos & $\begin{array}{l}\text { Insuficiência } \\
\text { respiratória } \\
\text { aguda }\end{array}$ \\
\hline $\begin{array}{l}\text { Andréjak et al., } \\
2013^{12}\end{array}$ & $\begin{array}{l}26 \text { pacientes não } \\
\text { sedados }\end{array}$ & $\begin{array}{l}22 \text { homens } \\
4 \text { mulheres }\end{array}$ & 67 anos & $\begin{array}{l}\text { Infecção } \\
\text { pulmonar/ } \\
\text { Exacerbação da } \\
\text { DPOC }\end{array}$ \\
\hline $\begin{array}{l}\text { Alexopoulou et } \\
\text { al., } 2007^{13}\end{array}$ & $\begin{array}{l}17 \text { pacientes, sedados } \\
\text { e não sedados. }\end{array}$ & $\begin{array}{l}11 \text { homens } \\
6 \text { mulheres }\end{array}$ & 64 anos & $\begin{array}{l}\text { Insuficiência } \\
\text { cardíaca/ Sepse }\end{array}$ \\
\hline $\begin{array}{l}\text { Toublanc et al., } \\
2007^{14}\end{array}$ & $\begin{array}{l}20 \text { pacientes não } \\
\text { sedados. }\end{array}$ & $\begin{array}{l}15 \text { homens } \\
5 \text { mulheres }\end{array}$ & 65 anos & $\begin{array}{l}\text { Infecção } \\
\text { pulmonar/ } \\
\text { Exacerbação da } \\
\text { DPOC }\end{array}$ \\
\hline $\begin{array}{l}\text { Roche-Campo et } \\
\text { al., } 2013^{15}\end{array}$ & $\begin{array}{l}15 \text { pacientes } \\
\text { traqueostomizados em } \\
\text { desmame, não } \\
\text { sedados. }\end{array}$ & $*$ & 68 anos & $*$ \\
\hline $\begin{array}{l}\text { Kondili et al., } \\
2012^{16}\end{array}$ & 12 pacientes em VM. & $\begin{array}{l}6 \text { homens } \\
6 \text { mulheres }\end{array}$ & 73 anos & $*$ \\
\hline $\begin{array}{l}\text { Bosma } \\
2007^{17}\end{array}$ et al. & $\begin{array}{l}13 \text { pacientes não } \\
\text { sedados }\end{array}$ & $\begin{array}{l}10 \text { homens } \\
3 \text { mulheres }\end{array}$ & 63 anos & Pneumonia \\
\hline
\end{tabular}

e a ventilação espontânea ${ }^{15}$. Neste estudo não foram encontrados despertares secundários a apneias centrais em modo PSV ${ }^{15}$, diferindo de outros estudos que demonstraram que pacientes ventilados neste modo apresentaram um número elevado de apneias centrais, culminando em um maior número de despertares, em comparação a pacientes ventilados em modo ventilação por pressão controlada $(\mathrm{PCV})^{4}$. Uma das causas de apneias centrais pode ser o uso inadequado de elevadas pressões de suporte além da demanda do paciente, promovendo aumento do volume 
Tabela 2. Detalhamento dos artigos incluídos

\begin{tabular}{ll}
\hline Autor & Método \\
\hline Alexopoulou et al., $\mathbf{2 0 1 3}^{\mathbf{2}}$ & $\begin{array}{l}\text { Avaliaram assincronias e qualidade do sono em } \\
\text { pacientes ventilados por PAV x PSV }\end{array}$ \\
\hline Cabello et al., $\mathbf{2 0 0 8}^{\mathbf{5}}$ & $\begin{array}{l}\text { Comparou a influência de } 3 \text { modos ventilatórios - } \\
\text { PCV, PSV e Smartcare } ~\end{array}$ \\
\hline Delisle et al. 20116 & Compararam o NAVA x PSV na arquitetura do sono. \\
\hline Andréjak et al., $\mathbf{2 0 1 3}^{\mathbf{1 2}}$ & $\begin{array}{l}\text { Avaliaram o efeito da PSV (PS=6cmH20) x PCV no } \\
\text { Sono, com foco no repouso muscular. }\end{array}$ \\
\hline Alexopoulou et al., $\mathbf{2 0 0 7}^{\mathbf{1 3}}$ & $\begin{array}{l}\text { Avaliaram se as oclusões inspiratórias realizadas na } \\
\text { PAV+ influenciam na qualidade do sono. } \\
\text { Compararam dois grupos: PAV+ e PSV. }\end{array}$ \\
\hline Toublanc, et al., $\mathbf{2 0 0 7}^{\mathbf{1 4}}$ & $\begin{array}{l}\text { Avaliaram o impacto do PCV x PSV com baixa pressão } \\
\text { inspiratória na qualidade do sono. }\end{array}$
\end{tabular}

Roche-Campo et al., 2013 ${ }^{15}$ Avaliaram o papel da VM no sono de pacientes em desmame de TQT, alternando VM e ventilação espontânea.

Kondili et al., $\mathbf{2 0 1 2}^{\mathbf{1 6}} \quad$ Avaliaram o efeito do propofol na qualidade do sono de pacientes em VM.

Bosma et al. 2007 $\mathbf{1 7}^{\mathbf{1 7}}$ Avaliaram as assincornias e etiologia da fragmentação do sono, comparando PAV+ x PSV.

PAV+: ventilação assistida proporcional plus. PSV: ventilação por pressão de suporte. PS: pressão de suporte. NREM: movimento não rápido dos olhos. REM: Movimento Rápido dos Olhos. VM: ventilação mecânica. TQT: traqueostomia. NAVA: Neurally adjusted ventilatory assist.

minuto, reduzindo o nível de CO2 na corrente sanguínea, levando a hipoventilação que cursa com apneia ${ }^{24}$.

Visando o descanso muscular pré-extubação, analisaram a alternância entre os modos PCV e PSV durante a noite, e demonstraram que no período "PCV" a qualidade de sono foi significativamente melhor do que quando em "PSV". Avaliaram também se a alternância para o modo PCV no período noturno atrasaria o desmame ventilatório, o que não se mostrou realidade, sendo todos os pacientes extubados na manhã seguinte ao protocolo. Concluíram então que períodos em PCV levam a descanso da 
musculatura respiratória, podendo então auxiliar na redução do tempo de desmame ventilatório ${ }^{12}$. Corroborando com outro estudo, que também demonstrou a melhora da qualidade do sono em modo PCV4.

Questiona-se também, a influência do modo ventilatório na consolidação do sono em pacientes graves. Avaliaram pacientes intubados, sendo submetidos a períodos de ventilação PCV, períodos de PSV e períodos de PSV acrescido de um espaço morto para tentar reduzir o número de apneias centrais. Perceberam que apenas $36 \%$ dos pacientes estudados alcançaram a fase REM do sono, e a eficácia maior do sono foi percebida quando em modo PCV e PSV+ adição do espaço morto. O volume minuto, o volume corrente e a variação do nível de CO2 expirado foi maior em PSV do que em PCV, e houve mais despertares em modo PSV do que quando na presença do espaço morto. Concluíram então que o modo PCV promove um sono mais eficaz, com menos despertares em comparação ao modo PSV, e que a adição do espaço morto foi capaz de reduzir o número de despertares pela redução do nível médio de $\mathrm{CO} 2$ expirado, corrigindo a hipocapnia reduzindo a incidência de apneias centrais ${ }^{4}$.

Estudo avaliou a qualidade do sono em pacientes não sedados utilizando a polissonografia e um questionário de autopercepção, comparando-os aos modos PCV e PSV com baixas pressões de suporte, visando a adequação do volume minuto afim de reduzir as apneias centrais. Todos os pacientes que realizaram o estudo conseguiram ser extubados na manhã seguinte, mostrando mais uma vez que 
períodos em modo PCV não foram capazes de atrasar o desmame da ventilação mecânica, além disso a qualidade do sono em ambas as avaliações se apresentou melhor quando em modo PCV ${ }^{14}$.

A fim de facilitar o desmame ventilatório, promovendo o descanso muscular, algumas UTIs utilizam sedativos durante a noite. Avaliaram o efeito da utilização do Propofol na qualidade do sono de pacientes mecanicamente ventilados sob modos assisto-controlados, e perceberam que o Propofol se apresentou como fator de risco direto para a apresentação de delirium, e promoveu a abolição, em quase todos os pacientes, da fase REM do sono. Concluiu-se então que o uso do Propofol para descanso da musculatura respiratória e melhora da qualidade do sono previamente ao desmame ventilatório não é seguro ${ }^{16}$.

Pode-se afirmar que, protocolos de sedação para pacientes em ventilação mecânica ainda precisam ser estudados. Deve-se levar em consideração os fatores ambientais como luminosidade, ruídos e movimentação da equipe e assincronias paciente-ventilador, antes da intervenção farmacológica ser escolhida ${ }^{25}$. Quando esta se faz necessária, é preciso avaliar as necessidades deste paciente pois no geral as medicações sedativas promovem alterações no ciclo circadiano e geram riscos para desenvolvimento de delirium ${ }^{26,27}$.

Com o advento dos novos modos ventilatórios, tem-se estudado sobre a influência destes na melhora da qualidade do sono. Ao avaliar essa interação paciente-ventilador em 14 
pacientes ventilados em modo PAV+ e PSV submetidos a polissonografia, observaram que todos os pacientes apresentaram arquitetura anormal do sono, 50\% do tempo total de sono ocorreu durante o $\mathrm{dia}^{2}$, mostrando inversão no ciclo circadiano observado anteriormente por outros autores $^{28,29}$. Também foi demonstrado que pacientes ventilados em modo PSV apresentaram um número maior de assincronias do que os ventilados em PAV+, demonstrando que a $\mathrm{PAV}+$ é capaz de melhorar a assincronia paciente ventilador, porém essa melhora não teve efeito significativo na qualidade do sono.

As assincronias podem não ser um dos principais determinantes da má qualidade do sono². Entretanto, alguns autores avaliaram o papel da interação paciente-ventilador na etiologia da interrupção do sono, comparando os modos PSV e PAV+, obtiveram melhora da qualidade do sono e redução do número de fragmentações em $\mathrm{PAV}+$, e a duração do sono foi semelhante nos dois modos. A pressão gerada pelo paciente para disparar o ventilador, e a pressão fornecida pelo ventilador, irão influenciar na qualidade do sono independentemente do modo ventilatório utilizado ${ }^{17}$.

Embora a PAV+ promova uma melhor interação paciente-ventilador, pelo aumento da capacidade do ventilador de suprir a demando do paciente, o ajuste adequado da pressão de suporte conforme o esforço do paciente pode auxiliar a melhora dessa interação, melhorando a qualidade do sono. Avaliou-se as microoclusões inspiratórias realizadas pelo modo $\mathrm{PAV}+$, para 
mensuração da mecânica respiratória; e sua interferência na qualidade do sono ${ }^{13}$. Os resultados mostraram melhora na eficiência e qualidade do sono quando comparado ao modo PSV, porém foi observado que em altas pressões ambos os modos ventilatórios podem proporcionar fragmentações do sono. Contudo, as micro-oclusões não tiveram influência na qualidade do sono ${ }^{13,17}$.

Ao comparar o uso do NAVA (Neurally Adjusted Ventilatory Assist) e o PSV, avaliaram o efeito do excesso de assistência, caracterizado por pressões de suporte elevadas em relação a necessidade do paciente, na qualidade do sono. Esse excesso de assistência não ocorreu no modo NAVA, justamente pela sua característica de disparo neural este modo foi capaz de eliminar os esforços ineficazes, reduzindo os despertares, apresentando maior tempo de sono REM, promovendo então uma melhor qualidade de sono frente ao uso do PSV6.

Ao comparar a influência de três modos ventilatórios e assincronias na qualidade do sono, utilizando SmartCare $\mathbb{R} / P S$, PSV e PCV, perceberam que $14 \%$ das fragmentações do sono durante o período de estudo foram relacionadas ao aumento de ruídos no ambiente ${ }^{5}$. O ruído é um dos vários fatores perturbadores do sono em pacientes em UTIs, sendo a conversa da equipe e os alarmes dos equipamentos utilizados neste ambiente, considerados os ruídos mais perturbadores ao sono ${ }^{30,31}$. Apesar de haver contradição entre alguns autores sobre a importância do ruído na qualidade do sono, sabe-se que as estratégias para 
minimizar esses ruídos são bem vindas quando tentamos proporcionar uma melhor qualidade de sono ${ }^{32}$.

Contudo, outros achados não obtiveram diferenças na eficiência e arquitetura do sono nos diferentes modos, contrapondo os achados anteriormente citados, levantando a necessidade de ajustes finos nos parâmetros ventilatórios, evitando a super-assistência que pode gerar apneias centrais e levar a fragmentação do sono ${ }^{5}$. É necessário que os parâmetros ventilatórios, e principalmente a pressão de suporte, sejam ajustados de forma personalizada levando em consideração que os ajustes realizados durante a vigília podem ser excessivos durante o sono quando há uma redução da demanda ventilatória ${ }^{33}$.

\section{CONCLUSÕES}

Os distúrbios do sono presentes na maioria dos pacientes em ventilação mecânica trazem prejuízos fisiológicos importantes, como a falha do desmame ventilatório, delirium, distúrbios psicológicos pós-internação, aumento do tempo de internação hospitalar, e consequentemente aumento dos custos.

Realizar a mudança do modo ventilatório de espontâneo para assisto-controlado durante o período da noite não se mostrou capaz de atrasar o desmame ventilatório, pelo contrário, evitou fadiga da musculatura respiratória, melhorou a qualidade e eficácia do sono e reduziu níveis de ansiedade. 
Mais importante do que mudança do modo ventilatório é o ajuste pormenorizado e personalizado dos parâmetros ventilatórios utilizados durante o período de sono. Sabe-se que altas pressões inspiratórias podem culminar em apneias centrais gerando despertares e fragmentação do sono. Esses ajustes ventilatórios se fazem mais eficazes nos novos modos ventilatórios como o PAV+ e o NAVA por suas características de avaliação da mecânica ventilatória que auxiliam no disparo da ventilação. A redução das assincronias ventilatórias pode auxiliar na redução da fragmentação do sono, e é sabido que estes, cada um com suas características, são capazes de eliminar os esforços ineficazes e reduzir o trabalho ventilatório.

O melhor método para monitorar o sono na UTI ainda não é bem estabelecido, além disso essa monitorização é dispendiosa e trabalhosa. As adequações ambientais junto aos ajustes ventillatorios auxiliam na promoção da qualidade do sono, permitindo descanso da musculatura respiratória, reduzindo o número de assincronias paciente-ventilador, podendo facilitar o desmame ventilatório, reduzindo o tempo de internação e evitando os efeitos deletérios da privação do sono. Portanto, além dos ajustes da ventilação mecânica, promover um ambiente adequado, com conscientização da equipe, melhora dos níveis de luminosidade e ruídos, auxilia no desenvolvimento de um sono mais restaurador.

Vale ressaltar que são necessários novos estudos que avaliem estratégias de adequação do ambiente para melhorar a qualidade do sono, além de comparações entre 
novos modos ventilatórios com adequação personalizada de volumes.

\section{REFERÊNCIAS}

1.Aurell, J, Emqvist D. Sleep in the surgical intensive care unit: continuous polygraphic recording of sleep in nine patients receiving postoperative care. $\mathrm{Br}$ Med J 1985;290:1029-32. https://doi.org/10.1136/bmj.290.6474.1029

2. Alexopoulou C, Kondili E, Plataki M, Georgopoulos D. Patientventilator synchrony and sleep quality with proportional assist and pressure support ventilation. Intensive Care Med 2013;39:1040-7. https://doi.org/10.1007/s00134-013-2850-y

3.Cooper AB, Gabor JY, Hanly PJ. Sleep in the critically ill patient. Semin Respir Crit Care Med 2001;22:153-64. https://doi.org/10.1055/s-2001-13829

4.Parthasarathy S, Tobin MJ. Effect of ventilator mode on sleep quality in critically ill patients. Am J Respir Crit Care Med 2002;166:1423-9. https://doi.org/10.1164/rccm.200209-9990C

5.Cabello B, Thille AW, Drouot X, Galia F, Mancebo J, d'Ortho MP, et al. Sleep quality in mechanically ventilated patients: comparison of three ventilatory modes. Crit Care Med 2008;36:1749-55. https://doi.org/10.1097/CCM.0b013e3181743f41

6.Delisle S, Ouellet P, Bellemare P, Tétrault JP, Arsenault P. Sleep quality in mechanically ventilated patients: comparison between NAVA and PSV modes. Ann Intensive Care 2011;1:42. https://doi.org/10.1186/2110-5820-1-42

7.Boesen $\mathrm{HC}$, Andersen JH, Bendtsen AO, Jennum PJ. Sleep and delirium in unsedated patients in the intensive care unit. Acta Anaesthesiol Scand 2016;60:59-68.

https://doi.org/10.1111/aas.12582

8. Haack M, Sanchez E, Mullington JM. Elevated inflammatory markers in response to prolonged sleep restriction are associated with increased pain experience in healthy volunteers. Sleep 2007;30:1145-52. https://doi.org/10.1093/sleep/30.9.1145

9.Durmer JS, Dinges DF. Neurocognitive consequences of sleep deprivation. Semin Neurol 2005;25:117-29.

https://doi.org/10.1055/s-2005-867080

10. White DP, Douglas NJ, Pickett CK, Zwillich CW, Weil JV. Sleep deprivation and the control of ventilation. Am Rev Respir Dis 1983;128:984-6. https://doi.org/10.1164/arrd.1983.128.6.984

11.Irwin M, McClintick J, Costlow C, Fortner M, White J, Gillin JC. Partial night sleep deprivation reduces natural killer and cellular imune responses in humans. FASEB J 1996;10:643-53. https://doi.org/10.1096/fasebj.10.5.8621064 
12. Andréjak C, Monconduit J, Rose D, Toublanc B, Mayeux $\mathrm{I}$, Rodenstein $\mathrm{D}$, et al. Does using pressure-controlled ventilation to rest respiratory muscles improve sleep in ICU patients? Respir Med 2013;107:534-41. https://doi.org/10.1016/j.rmed.2012.12.012

13.Alexopoulou C, Kondili E, Vakouti E, Klimathianaki M, Prinianakis G, Georgopoulos D. Sleep during proportional-assist ventilation with load-adjustable gain factors in critically ill patients. Intensive Care Med 2007;33:1139-47. https://doi.org/10.1007/s00134-007-0630-2

14.Toublanc B, Rose D, Glérant JC, Francois G, Mayeux I, Rodenstein $D$, et al. Assist-control ventilation vs. low levels of pressure support ventilation on sleep quality in intubated ICU patients. Intensive Care Med 2007;33:1148-54. https://doi.org/10.1007/s00134-007-0659-2 15.Roche-Campo F, Thille AW, Drouot X, Galia F, Margarit L, CórdobaIzquierdo $A$, et al. Comparison of sleep quality with mechanical versus spontaneous ventilation during weaning of critically ill tracheostomized patients. Crit Care Med 2013;41:1637-64. https://doi.org/10.1097/CCM.0b013e318287f569

16.Kondili E, Alexopoulou C, Xirouchaki N, Georgopoulos D. Effects of propofol on sleep quality in mechanically ventilated critically ill patients: a physiological study. Intensive Care Med 2012;38:16406. https://doi.org/10.1007/s00134-012-2623-z

17.Bosma K, Ferreyra G, Ambrogio C, Pasero D, Mirabella L, Braghiroli $A$, et al. Patient-ventilator interaction and sleep in mechanically ventilated patients: pressure support versus proportional assist ventilation. Crit Care Med 2007;35:1048-54.

https://doi.org/10.1007/s00134-012-2623-z

18. Freedman NS, Gazendam J, Levan L, Pack AI, Schwab RJ. Abnormal sleep/wake cycles and the effect of environmental noise on sleep disruption in the intensive care unit. Am J Respir Crit Care Med 2001;163:451-7. https://doi.org/10.1164/ajrccm.163.2.9912128

19. Friese RS, Diaz-Arrastia R, McBride D, Frankel H, Gentilello LM. Quantity and quality of sleep in the surgical intensive care unit: are our patients sleeping? J Trauma 2007;63:1210-4.

https://doi.org/10.1097/TA.0b013e31815b83d7

20.Drouot X, Roche-Campo F, Thille AW, Cabello B, Galia F, Margarit $L$, et al. A new classification for sleep analysis in critically ill patients. Sleep Med 2012;13:7-14.

https://doi.org/10.1016/j.sleep.2011.07.012

21. Watson PL, Pandharipande P, Gehlbach BK, Thompson JL, Shintani AK, Dittus BS, et al. Atypical sleep in ventilated patients: empirical electroencephalography findings and the path toward revised ICU sleep scoring criteria. Crit Care Med 2013;41:1958-67. https://doi.org/10.1097/CCM.0b013e31828a3f75

22. Thille AW, Reynaud F, Marie D, Barrau S, Rousseau L, Rault C, et al. Impact of sleep alterations on weaning duration in mechanically ventilated patients: a prospective study. Eur Respir J 2018;51:1702465. https://doi.org/10.1183/13993003.02465-2017 
23. Chen HI, Tang YR. Sleep loss impairs inspiratory muscle endurance. Am Rev Respir Dis 1989;140:907-

9. https://doi.org/10.1164/ajrccm/140.4.907

24. Meza S, Mendez M, Ostrowski M, Younes M. Susceptibility to periodic breathing with assisted ventilation during sleep in normal subjects. J Appl Physiol 1998;85:1929-40.

https://doi.org/10.1152/jappl.1998.85.5.1929

25. Weinhouse GL, Watson PL. Sedation and Sleep Disturbances in the ICU. Crit Care Clin 2009;25:539-49.

https://doi.org/10.1016/j.ccc.2009.04.003

26. Andersen JH, Boesen HC, Skovgaard Olsen K. Sleep in the intensive care unit measured by polysomnography. Minerva Anestesiol 2013;79:804-15.

https://www.minervamedica.it/en/getfreepdf/DoMild\%252FyALTG8iF VSyqpVjBEN2pxmeXZnfOPnQqC\%252BjsSZdfDmeVjJb0X54mgr7zKW o\%252FYHHDnhzv\%252FtZowynAgOw\%253D\%253D/R02Y2013N07A 0804.pdf

27.Alexopoulou C, Kondili E, Diamantaki E, Psarologakis C, Kokkini $\mathrm{S}$, Bolaki $\mathrm{M}$, et al. Effects of dexmedetomidine on sleep quality in critically ill patients. Anesthesiology 2014;121:801-7. https://doi.org/10.1097/ALN.0000000000000361

28. Gehlbach BK, Chapotot F, Leproult R, Whitmore H, Poston J, Pohlman $M$, et al. Temporal disorganization of circadian rhythmicity and sleep-wake regulation in mechanically ventilated patients receiving continuous intravenous sedation. Sleep 2012;35:1105-14. https://doi.org/10.5665/sleep.1998

29.Telias I, Wilcox ME. Sleep and Circadian Rhythm in Critical Illness. Crit Care 2019;23:82. https://doi.org/10.1186/s13054-019-2366-0 30.Elliott R, McKinley S, Cistulli P, Fien M. Characterisation of sleep in intensive care using 24-hour polysomnography: an observational study. Crit Care 2013;17:R46. https://doi.org/10.1186/cc12565

31.Gabor JY, Cooper AB, Crombach SA, Lee B, Kadikar N, Bettger $\mathrm{HE}$, et al. Contribution of the intensive care unit environment to sleep disruption in mechanically ventilated patients and healthy subjects. Am J Respir Crit Care Med 2003;167:708-15.

https://doi.org/10.1164/rccm.2201090

32.Xie $\mathrm{H}$, Kang J, Mills $\mathrm{GH}$. Clinical review: The impact of noise on patients' sleep and the effectiveness of noise reduction strategies in intensive care units. Crit Care 2009;13:208. https://doi.org/10.1186/cc7154

33. Nakayama H, Smith CA, Rodman JR, Skatrud JB, Dempsey JA. Effect of ventilatory drive on carbon dioxide sensitivity below eupnea during sleep. Am J Respir Crit Care Med 2002;165:1251-60. https://doi.org/10.1164/rccm.2110041 